\title{
Recent Advances in the Diagnosis and Treatment of Influenza Pneumonia
}

\author{
Lucia Marzoratti • Hernán A. Iannella • \\ Victoria Fernández Gómez • Sandra B. Figueroa
}

Published online: 3 April 2012

(C) The Author(s) 2012. This article is published with open access at Springerlink.com

\begin{abstract}
A potentially fatal complication of influenza infection is the development of pneumonia, caused either directly by the influenza virus, or by secondary bacterial infection. Pneumonia related to the 2009 influenza A pandemic was found to be underestimated by commonly used pneumonia severity scores in many cases, and to be rapidly progressive, leading to respiratory failure. Confirmation of etiology by laboratory testing is warranted in such cases. Rapid antigen and immunofluorescence testing are useful screening tests, but have limited sensitivity. Confirmation of pandemic H1N1 influenza A infection can only be made by real-time reverse-transcriptase polymerase chain reaction (rRT-PCR) or viral culture. The most effective preventive measure is annual influenza vaccination in selected individuals. Decisions to administer antiviral medications for influenza treatment or chemoprophylaxis should be based upon clinical and epidemiological factors, and should not be delayed by confirmatory laboratory testing results. Neuraminidase inhibitors (NI) are the agents of choice.
\end{abstract}

L. Marzoratti $(\bowtie) \cdot$ V. F. Gómez $\cdot$ S. B. Figueroa CEMIT (Centro Médico Investigadores Tucumán), San Miguel de Tucumán, Tucumán, Argentina

e-mail: luciamarzoratti@gmail.com

V. F. Gómez

e-mail: fvictoriarosa@yahoo.com

S. B. Figueroa

e-mail: sbfigueroa@hotmail.com

H. A. Iannella

Pulmonary Medicine Division, Hospital de Clínicas,

Universidad de Buenos Aires,

Buenos Aires, Argentina

e-mail: hiannella@fmed.uba.ar
Keywords Influenza pneumonia - Diagnosis - Treatment . Prevention $\cdot$ Swine influenza $\cdot$ Avian influenza $\cdot \mathrm{H} 1 \mathrm{~N} 1$. Pandemic

\section{Introduction}

Influenza is acute respiratory illness caused by influenza A or B viruses with seasonal circulation during the winter months. However, outbreaks of novel recombinant strains that take place in animals have produced, along the years, many worldwide outbreaks with serious public health issues. Although normally a self-limited process in the general population, high risk groups for complications and death have been identified (Table 1). During an outbreak, in otherwise healthy subjects the diagnosis of influenza infection can be made confidently based on clinical manifestations alone. However, in certain situations, such as sporadic cases, in patients at an increased risk for complications, or in hospitalized patients with severe pulmonary compromise, confirmation of etiology by laboratory testing is required to guide treatment and for surveillance purposes $[1 \bullet, 2 \bullet \cdot$. A potentially fatal complication of influenza infection is the involvement of the lower respiratory tract caused directly by the influenza virus, and the development of secondary bacterial pneumonia. Novel recombinant influenza A strains carry the risk for more severe disease and have the potential to cause widespread illness and a large number of deaths, regardless of age or previous health status. Examples of this are the H5N1 "avian" influenza A outbreak since 2004, and the more recent H1N1 "swine" influenza A pandemic in 2009, both of which have prompted the development of quick and reliable laboratory test in an effort to optimize their management and reduce morbidity and mortality. In 
Table 1 Persons at higher risk for influenza complications

\author{
Children $<2$ years \\ Adults $\geq 65$ years \\ Women who are pregnant or postpartum (within 2 weeks after \\ delivery) \\ Individuals with chronic medical conditions \\ Pulmonary disease (including asthma) \\ Cardiovascular disease (except isolated hypertension) \\ Active malignancy \\ Chronic renal insufficiency \\ Chronic liver disease \\ Diabetes mellitus \\ Hemoglobinopathies (including sickle cell disease) \\ Immunosuppression ${ }^{1}$ \\ Any neurologic condition that can compromise handling of \\ respiratory secretions ${ }^{2}$ \\ Native Americans and Alaska Natives \\ Morbidly obese persons (body-mass index $\geq 40$ ) \\ Residents of nursing homes and other chronic-care facilities
}

(1) Including HIV infection (particularly if CD4 $<200$ cells/microL), organ or hematopoietic cell transplantation and inflammatory disorders treated with immunosuppressants

(2) Including disorders of the brain, spinal cord, peripheral nerve, and muscle such as cerebral palsy, seizure disorders, stroke, intellectual disability, moderate to severe developmental delay, muscular dystrophy, or spinal cord injury

Adapted from the recommendations of the Advisory Committee on Immunization Practices (ACIP) [40••]

this article, we review the latest available data for the diagnosis of influenza lower respiratory tract infection, as well as for treatment and prevention strategies.

\section{Clinical and Radiological Diagnosis}

Signs and symptoms of upper and/or lower respiratory tract infection, along with systemic involvement in the form of fever, myalgia, and headache, are usually the main presenting features of the disease. In the context of an outbreak, otherwise healthy subjects presenting with a self-limited acute febrile respiratory illness usually require no further diagnostic procedures. In two retrospective studies that examined which clinical signs and symptoms are most predictive of influenza infection in patients with influenza-like illness, cough and fever were the only symptoms significantly associated with a positive PCR test for influenza [3, 4]. In another study, no isolated symptom or sign was able to accurately predict influenza infection, though the absence of fever, cough and nasal congestion significantly decreased its likelihood [5]. In general, patients diagnosed with pandemic H1N1 influenza A virus had similar signs and symptoms compared to those with seasonal influenza. However, these patients had gastrointestinal manifestations more frequently $[6,7]$, were more likely to have pneumonia [8], and also had higher rates of extrapulmonary complications, intensive care unit admission, and death [9].

Pneumonia is the most frequent and severe complication of influenza, most commonly presenting in high risk patients (Table 1). Primary influenza pneumonia represents direct lung involvement by influenza virus, and should be suspected in non-resolving influenza infections. Typically, primary influenza pneumonia presents in chest x-rays with bilateral reticular or reticulonodular opacities. Less frequently, focal areas of consolidation can be seen, particularly in the lower lobes. High-resolution computed tomography may show ground glass opacities with or without multifocal peribronchovascular and subpleural consolidation [10].

The cytopathic effect of the influenza virus on the tracheobronchial epithelium may predispose to secondary bacterial pneumonia $[11,12]$. Secondary bacterial pneumonia must be suspected whenever there is an exacerbation of fever and respiratory symptoms after initial improvement in a patient diagnosed with acute influenza. Leukocytosis, instead of a normal or low white blood cell count, and lobar consolidation on chest imaging, instead of the diffuse pattern that is typical of viral pneumonia, are also suggestive [13].

In an observational study of 543 hospitalized patients with H1N1 influenza A infection in Spain, $43 \%$ of the 243 patients in which chest radiographs were performed had pneumonia, $83 \%$ of the 210 patients who had microbiologic confirmation had primary influenza pneumonia, and the remaining $17 \%$ had concomitant secondary bacterial pneumonia. Bilateral pneumonia occurred in $48.3 \%$ of patients; Streptococcus pneumoniae being the most frequent pathogen [14]. Several reports have identified methicillinresistant Staphylococcus aureus (MRSA) as the etiologic agent for severe community acquired pneumonia (CAP) in otherwise healthy young patients with influenza [15-17]. In another study that investigated the incidence of communityacquired MRSA pneumonia in H1N1 influenza patients, 50 patients of 4491 (1\%) laboratory-confirmed pandemic influenza A $(\mathrm{H} 1 \mathrm{~N} 1)$ cases had a bacterial respiratory tract pathogen. The most commonly cultured organisms were $S$. pneumoniae (16 patients), S. aureus (13 patients) and Haemophilus influenzae (9 patients); MRSA was detected in only 2 patients [18]. In contrast, among 838 children and adolescents admitted to 35 intensive care units in the U.S. with confirmed or probable severe H1N1 influenza A infection, $48 \%$ of the 71 patients with suspected diagnosis of early $S$. aureus coinfection had MRSA [19].

Non-seasonal influenza infections have specific clinical manifestations. Pneumonia related to the 2009 H1N1 influenza A pandemic was also found in many cases to be rapidly progressive, leading to respiratory failure and ARDS [20•, 21•]. Additionally, the risk for complications and death due to that 
pandemic influenza was found to be underestimated by commonly used pneumonia severity scores [22•, 23]. Avian influenza (H5N1) frequently presents as severe primary pneumonia that often progresses rapidly to the acute respiratory distress syndrome (ARDS), having caused high rates of death, especially among infants and young children in Southeast Asian countries [24].

\section{Laboratory Testing}

In certain situations, confirmation of etiology by laboratory testing is required in order to guide the initiation and duration of antiviral therapy, and for the implementation of infection control measures and surveillance. Other benefits of influenza virus detection are the reduction of inappropriate antibiotic use, decreased length of stay in emergency departments, and fewer additional laboratory studies, all leading to a reduction in health care costs $[1 \bullet \bullet]$. The Centers for Disease Control and Prevention (CDC) and the Infectious Diseases Society of America (IDSA) have published guidelines to better define patients who should undergo influenza testing $[1 \bullet \bullet, 2 \bullet \cdot]$. The available methods include immunological techniques (i.e. rapid antigen-based tests, immunofluorescence assays, serologic testing), molecular techniques (i.e. reverse-transcriptase polymerase chain reaction [RT-PCR]), and microbiological techniques (i.e. viral cultures). While RT-PCR has the highest sensitivity and specificity, rapid antigen and immunofluorescence testing, though very useful as initial screening tests, are considerably less sensitive. Besides, the sensitivity and specificity of any of these techniques will vary depending on the laboratory equipment, personnel expertise, the timing of rec-

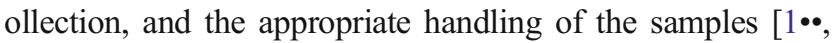
$2 \bullet \bullet]$. Respiratory specimens can be obtained by many different methods including throat swabs, nasal aspirates, and nasopharyngeal swabs, aspirates and washing [2••]. In mechanically ventilated subjects, more invasive maneuvers such as endotracheal aspirates and bronchoscopic or non-bronchoscopic bronchoalveolar lavage (BAL) may be required in order to obtain adequate lower respiratory tract samples. While rapid antigen tests, immunofluorescence and RT-PCR all can yield results quick enough to guide point-of-care clinical decisionmaking, serologic tests and viral cultures provide retrospective diagnosis, availability only in reference laboratories, and usefulness when confirming screening test. For this reason, they are normally reserved for epidemiological and research purposes (Table 2).

\section{Rapid Influenza Diagnostic Tests}

Rapid influenza diagnostic test (RIDT) are designed to detect influenza $\mathrm{A}$ and $\mathrm{B}$ nucleoproteic antigens in respiratory specimens, with results expressed qualitatively as positive or negative in no more than $15 \mathrm{~min}$. Many different FDAapproved tests are available; while some assays are capable of distinguishing between influenza A or B viruses, none can distinguish between pandemic and seasonal strains of influenza A $[1 \bullet \cdot, 2 \bullet \cdot$. Compared to reference methods (i.e. RT-PCR and viral culture), they have low sensibility (10\% to $80 \%)$ and high specificity ( $95 \%$ to $100 \%$ ), and there is also great variability between the different commercially available kits [25-27]. Sensitivity appears to be somewhat lower for influenza B than that seen for influenza A [26]. Also, the reported sensitivity for rapid tests is higher for nasopharyngeal samples than for throat swabs [27]. There are two main factors that influence RIDT's negative predictive value. First, negative results obtained during periods of high viral activity in the community are more likely to be false negatives; alternatively, false positives, though much less frequent, are more likely during periods of less viral circulation. Second, respiratory samples collected within the first 48 to $72 \mathrm{~h}$ of symptom onset positively influence RIDT's sensitivity. For these reasons, when rapid tests are negative, confirmation by means of RTPCR or viral culture should be considered $[1 \bullet \bullet, 2 \bullet \bullet]$. Compared to RT-PCR, the sensitivity of RIDTs for detecting novel influenza A (H1N1) was equal to or lower than the sensitivity to detect seasonal influenza viruses [29-32], so RIDT results need to be interpreted with caution when evaluating patients suspected of having pandemic H1N1 influenza A.

\section{Immunofluorescence}

Direct (DFA) or indirect (IFA) immunofluorescence antibody staining techniques are capable of detecting influenza $A$ and $B$ viruses, and distinguish the viruses from each other as well as from other respiratory viruses $[1 \bullet \bullet, 2 \bullet \bullet$. They have levels of sensitivity and specificity that come close to those of RT-PCR [28, 33], and results are often available in a few hours $[1 \bullet, 2 \bullet \bullet]$. Although these tests have improved sensitivity over RIDT, they are more technically complex and require expertise in obtaining quality respiratory samples (Table 2).

\section{Reverse-Transcriptase Polymerase Chain Reaction}

This is the reference influenza detection method and has the highest sensitivity and specificity $[34,35]$. Several modalities of RT-PCR have been designed: conventional gel-based PCR (cRT-PCR), multiplex PCR (mRT-PCR), and real-time RT-PCR (rRT-PCR). They can differentiate between influenza types (A or B) and subtypes (including pandemic H1N1 influenza and avian $\mathrm{H} 5 \mathrm{~N} 1$ influenza), and results are available in 2-6 h (although due to transportation of batched specimens to reference centers for processing, it may take longer for 
Table 2 Influenza testing methods

\begin{tabular}{lllllll}
\hline Method & Test Time & Specimen & Sensitivity & Specificity & $\begin{array}{c}\text { Distinguishes influenza } \\
\text { A from B }\end{array}$ & Influenza A subtypes \\
\hline RITD & $15 \mathrm{~min}$. & Respiratory samples $^{1}$ & + & +++ & yes $^{2}$ & no \\
Immuno-fluorescence $^{3}$ & $1-4 \mathrm{~h}$. & Respiratory samples $^{1}$ & ++ & +++ & yes & no \\
RT-PCR $^{4}$ & $1-6 \mathrm{~h}$. & Respiratory samples $^{1}$ & +++ & ++++ & yes & yes \\
Viral culture $^{5}$ & $1-10 \mathrm{~d}$. & Respiratory samples $^{1}$ & ++ & +++++ & & yes \\
Serologic tests & $2 \mathrm{w}$. & Serum $^{6}$ & $\mathrm{n} / \mathrm{a}$ & $\mathrm{n} / \mathrm{a}$ & yes & \\
\hline
\end{tabular}

$R I D T$ rapid influenza diagnostic tests, $R T-P C R$ reverse-transcriptase polymerase chain reaction

(1) Appropriate respiratory samples vary for each method and should be obtained according to the manufacturer's specifications

(2) Some commercially-available RIDT distinguish between influenza A and B while others do not

(3) Direct (DFA) or indirect (IFA) antibody staining

(4) Conventional gel-based PCR, real-time RT-PCR, and multiplex PCR

(5) Rapid viral cultures (shell vials) can yield results in 1-3 days, compared to conventional isolation in cell culture (3-10 days)

Adapted from the IDSA and CDC guidelines $[1 \bullet \bullet, 2 \cdot \bullet]$

results to be available). During the 2009 H1N1 influenza A pandemic, it became clear that rapid case identification was essential for timely management of patients and for adequate public health actions to be taken. To answer to this threat, the CDC optimized the previously developed rRT-PCR procedures for detection of the A/H1N1 2009 pandemic influenza virus [36•]. Yang et al. compared the performance of 12 rRTPCR primer-probe sets designed for detecting the hemagglutinin (HA) or the neuraminidase (NA) gene of the pandemic influenza A/H1N1 2009 virus, using the primer-probe set developed at the CDC as reference. They found that although all primer-probe sets had specificity levels as high as $98.4 \%$ to $100 \%$, some of the primer-probe sets had better specificity, sensitivity, and amplification efficiency than others, and that a combination of primer-probe sets targeted to the HA and NA genes had higher detection sensitivity than those targeting HA or NA individually [37]. In another study, Lam et al. showed that although rRT-PCR assays can be 10-fold more sensitive than cRT-PCR, newly developed cRT-PCR assays targeting the HA gene are a reliable alternative for laboratories where a rRT-PCR machine is not available [38]. Sensitivity and specificity of these assays also depend on the type of respiratory sample employed. For example, in a Spanish study that assessed the utility of rRT-PCR for the diagnosis of the novel influenza $\mathrm{A} / \mathrm{H} 1 \mathrm{~N} 1$ virus, the authors reported that the diagnostic yield of combined nose and throat swabs was higher than that of nasopharyngeal aspirates [39].

\section{Viral Cultures and Serologic Tests}

Even the fastest viral cultures techniques can take days to demonstrate influenza cytopathic effects. Since they are not suitable for initial clinical management, their utility during an influenza outbreak is to confirm some negative test results from RIDT and immunofluorescence. Viral cultures also provide information about circulating influenza strains and its subtypes; this information is required for next season vaccine production, for surveillance of the emergence of new influenza A strains, and for the detection of antiviral resistance. Similarly, serologic tests (i.e., hemagglutinin inhibition, ELISA, complement-fixation) that demonstrate a four-fold increase in serum antibody titers between acute and convalescence phases of the disease, are only useful for retrospective diagnosis or research purposes (Table 2).

\section{Treatment and Prevention}

Annual immunization is the most important preventive measure $[40 \bullet \bullet$. However, two classes of antiviral drugs are available and play an important role in the treatment and prevention of influenza [41••]: the neuraminidase inhibitors (NI), oseltamivir and zanamivir, which are active against both influenza A and B viruses; and the M2 inhibitors, amantadine and rimantadine, which are active against all influenza A strains, but have no activity against influenza B viruses. In general, the duration for therapy with an NI is 5 days, and with the M2 inhibitors is three to 5 days.

\section{Pharmacology}

NI are sialic acid analogs that competitively inhibit neuraminidase on the surface of both influenza A and B, thus interfering with the release of virus from infected cells. Oseltamivir phosphate is an orally bioavailable prodrug that is rapidly absorbed from the gastrointestinal tract and is converted by hepatic esterases to the active metabolite, oseltamivir carboxylate. It has an elimination half-life of approximately $8 \mathrm{~h}$, primarily 
through the kidneys, and dose reduction is recommended for patients with an estimated creatinine clearance of less than $30 \mathrm{~mL} / \mathrm{min}$. Approximately $15 \%$ of inhaled zanamivir reaches the bronchi and lungs. Excretion is primarily renal, but given its limited systemic bioavailability, there is no need to modify the dose in patients with renal insufficiency. The pulmonary half-life is $2.8 \mathrm{~h}$. The most common toxicities reported with NI have been nausea and vomiting (approximately $15 \%$ of patients). These side effects are usually mild and limited to the first days of treatment, although more serious side effects have been described. Oseltamivir has been linked to selfinjury and delirium in pediatric populations, although no causal association could be demonstrated $[42,43]$. The use of inhaled zanamivir has been associated with bronchospasm, sometimes severe or fatal, particularly in patients with underlying airways disease such as chronic obstructive pulmonary disease (COPD) or asthma [44].

Amantadine and rimantadine target the M2 protein of influenza $\mathrm{A}$, which forms a proton channel in the viral membrane that is essential for viral replication. Amantadine is primarily excreted unchanged in the urine. In patients older than 65 years and in those with an estimated creatinine clearance of less than $50 \mathrm{~mL} / \mathrm{min}$, the daily dose should be reduced. Rimantadine is extensively metabolized in the liver, and dose reduction is recommended for patients with severe hepatic dysfunction, renal failure (creatinine clearance $<10 \mathrm{~mL} / \mathrm{min}$ ), and the elderly. Central nervous system (CNS) side effects are well described with amantadine, particularly in elderly patients. In comparison, rimantadine is associated with a considerably reduced rate of CNS side effects [45].

\section{Clinical Outcomes}

In mild to moderate uncomplicated disease, the reported benefits of early treatment $(<48 \mathrm{~h}$ of symptom onset) with NI have been a shorter duration and severity of flu-like symptoms, and a reduced duration of viral shedding [46-49]. More importantly, several trials and a few systematic reviews have shown that treatment with NI may reduce illness severity and the rate of lower respiratory tract complications [47, 50-52]. In a meta-analysis of 10 randomizedcontrolled trials, Kaiser et al. found that therapy with oseltamivir was effective in reducing the incidence of influenzarelated lower respiratory tract complications that required antibiotic use (4.6\% for oseltamivir vs. $10.3 \%$ for placebo, $\mathrm{P}<0.001$ ), independent of the presence of risk factors for complications [51]. More recently, Hernán et al. conducted another meta-analysis of 11 controlled trials, most of which were included in the previous meta-analysis by Kaiser et al. They found that treatment with oseltamivir significantly reduced influenza-related lower respiratory tract complications by $37 \%$ (CI $95 \%$ [18-52]) [52]. NI have also been reported to reduce the duration of hospitalization in severely-ill cases [53], and also to reduce influenza-related mortality [54]. During the 2009 H1N1 Influenza A pandemic, several observational studies of hospitalized and critically-ill patients reported that treatment with oseltamivir reduced disease severity, complications and mortality [55-57]. In a Chinese study of 1291 patients with confirmed H1N1 influenza A infection, oseltamivir reduced the risk of developing pneumonia, even when administered after the first $48 \mathrm{~h}$ of symptom onset (OR 0.12 , $95 \%$ CI [0.08-0.18]) [58]. In another retrospective study of 304 hematopoietic stem cell transplant recipients with influenza infection, 161 patients had H1N1 influenza A confirmed infection, and both early and delayed administration of antiviral therapy was shown to be beneficial in terms of decreased risk of lower respiratory tract compromise $(\mathrm{OR}=0.04,95 \%$ CI [0-0.2] vs. $\mathrm{OR}=0.14,95 \% \mathrm{CI}[0-0.7])$ [59]. An intravenous form of zanamivir is under development, and was made available during the $2009 \mathrm{H} 1 \mathrm{~N} 1$ influenza A pandemic for severely ill patients with highly suspected or confirmed oseltamivir-resistant infection that could not tolerate inhaled zanamivir. Several studies reported favorable outcomes with the use of IV zanamivir [60-63].

During the 2009 swine influenza pandemic, the FDA briefly authorized the emergency use of peramivir, an investigational NI that is administered intravenously [64]. In one study, a single dose of $300 \mathrm{mg}$ or $600 \mathrm{mg}$ of IV peramivir significantly reduced the time to alleviation of symptoms compared with placebo (HR 0.68 and 0.67 for the $300 \mathrm{mg}$ and $600 \mathrm{mg}$ doses respectively) [65]. Laninamivir octanoate, another long acting NI in development, was non-inferior to a five-day course of oral oseltamivir in adults with seasonal influenza in one study [66]. Experience with avian influenza H5N1, which has caused sporadic cases since 2004 with an elevated mortality rate, is much more limited and recommendations for its treatment are in most cases extrapolated from trials of seasonal influenza. There are, however, reports that suggest reduced severity and mortality with the administration of oseltamivir in patients with avian influenza [67-69]. In patients with pneumonia or with clinical failure to standard regime with oseltamivir, a higher dose of $150 \mathrm{mg}$ bid for 10 days should be considered [24, 67]. In regions with adamantine-susceptible strains, combination therapy with a NI and an adamantine may also be considered with pneumonia or clinical worsening [24]. Therapy with oseltamivir should be administered even in the late course of influenza A (H5N1) infection since viral replication is more prolonged than with seasonal influenza [24].

\section{Indications for Treatment}

The United States Advisory Committee on Immunization Practices (ACIP) and the CDC have recently published 
updated guidelines for the treatment of patients with confirmed or suspected influenza virus infections caused by either pandemic H1N1 or seasonal strains $[41 \bullet \bullet, 70 \bullet \bullet$. According to these recommendations, those individuals with severe disease (requiring hospitalization or with evidence of lower respiratory tract infection) or those at high risk for complications (Table 1) should receive antiviral therapy even after $48 \mathrm{~h}$ of symptom onset. Adults with mild illness without high risk conditions who are younger than 65 years of age do not require treatment. If such individuals present within the first $48 \mathrm{~h}$ of illness, antiviral treatment can be considered in order to reduce the duration of illness. In all cases, decisions to administer antiviral medications for influenza treatment should be based on clinical and epidemiologic grounds, and never be delayed because of confirmatory laboratory tests $[41 \bullet \cdot]$. The usual dosing of oseltamivir for the treatment of influenza is $75 \mathrm{mg}$ orally twice daily and of zanamivir is $10 \mathrm{mg}$ ( 2 inhalations) twice daily. The recommended duration for antiviral treatment is 5 days, although longer treatment courses for patients who fail to improve after 5 days of treatment can be considered $[70 \bullet$. Also, doubling the dose of oseltamivir to $150 \mathrm{mg}$ orally twice daily has been suggested to be beneficial for some severely ill patients with H5N1 avian influenza [24, 67]. The FDA licensed oseltamivir for use in children 1 year of age and older in a liquid formulation at a dosage of $2 \mathrm{mg} / \mathrm{kg} /$ dose twice daily for 5 days. Zanamivir is not approved for use in children under 5. Oseltamivir and zanamivir are Pregnancy Category $\mathrm{C}$ drugs, but since influenza causes more severe disease and an increased rate of mortality among pregnant women, they should receive antiviral therapy with oseltamivir when indicated, since the potential benefit outweighs the theoretical risk to the fetus (Table 1).

\section{Resistance}

Although oseltamivir-resistant seasonal H1N1 influenza A viruses have been identified since 2007 (the H274Y mutation) [71], in 2009 the CDC reported that most circulating strains of the novel H1N1 influenza A virus were sensitive to the NI, oseltamivir and zanamivir, but that nearly all strains were resistant to the amantadines $[41 \bullet \bullet, 72]$. Consequently, the ACIP has advised against the use of M2 inhibitors for treatment of influenza, except in selected circumstances [41••].

\section{Additional Management Strategies}

It is recommended that patients with pandemic H1N1 influenza A who develop pneumonia be treated empirically for CAP according to published evidence-based guidelines, given the risk of secondary bacterial pneumonia [73]. In the presence of profound hypoxemia that has been refractory to routine mechanical ventilation, salvage therapies include neuromuscular blockade, inhaled nitric oxide, high-frequency oscillatory ventilation, extracorporeal membrane oxygenation (ECMO), and prone positioning ventilation $[74 \cdot, 75]$. Corticosteroids should not be used routinely, but may be considered for septic shock with suspected adrenal insufficiency requiring vasopressors [76]. Therapy for influenza-associated ARDS should be based upon published evidence-based guidelines for sepsis-associated ARDS, specifically including lung protective mechanical ventilation strategies [76].

\section{Prevention}

The CDC recommends routine annual influenza vaccination for all persons 6 months of age and older. When vaccine supply is limited, vaccination efforts should focus on those groups with health conditions associated with increased risk of influenza complications [40••]. Antiviral drugs should not be used as a substitute for influenza vaccination. Their adjunctive use is appropriate in certain targeted populations at high risk for complications of influenza who are close contacts with suspected or confirmed cases [Table 1]. Postexposure prophylaxis should only be used when antivirals can be started within $48 \mathrm{~h}$ of the most recent exposure. Recommended duration is 7 days after exposure, and the CDC recommends a minimum of 2 weeks for control of influenza outbreaks in long-term care facilities (i.e., nursing homes with elderly) and hospitals [70••]. The choice to offer post-exposure prophylaxis to otherwise healthy unvaccinated adults should be weighed against the risk of promoting antiviral drug resistance $[41 \bullet \bullet, 1 \bullet \cdot]$.

\section{Conclusions}

A potentially fatal complication of influenza infection is the involvement of the lower respiratory tract caused directly by the influenza virus, and the development of secondary bacterial pneumonia. In these cases, and in patients who are at an increased risk for influenza infection complications, confirmation of etiology by laboratory testing is required in order to guide the initiation and duration of antiviral treatment, and for the implementation of infection control measures and surveillance. Recently, the emergence of novel influenza A strains that carry the risk for more severe disease regardless of age or previous health status, has prompted the development of quick and reliable laboratory tests in an effort to optimize their management and reduce morbidity and mortality. Pneumonia related to the 2009 influenza A pandemic was found in many cases to be rapidly progressive, leading to respiratory failure which in 
many cases was underestimated by commonly used pneumonia severity scores. Given the limited sensitivity of RIDT and immunofluorescence assays, confirmation of pandemic H1N1 influenza A infection can only be made by rRT-PCR or viral culture. Although annual immunization is the most important preventive measure, NI are the agents of choice for chemoprophylaxis in selected high risk patients, and for treatment. Treatment with NI beyond $48 \mathrm{~h}$ of symptoms should be considered only for patients with severe disease.

Disclosure No potential conflicts of interest relevant to this article were reported.

Open Access This article is distributed under the terms of the Creative Commons Attribution License which permits any use, distribution, and reproduction in any medium, provided the original author(s) and the source are credited.

\section{References}

Papers of particular interest, published recently, have been highlighted as:

- Of importance,

•- Of major importance

1. • Harper SA, Bradley JS, Englund JA, et al.: Seasonal Influenza in Adults and Children-Diagnosis, Treatment, Chemoprophylaxis, and Institutional Outbreak Management: Clinical Practice Guidelines of the Infectious Diseases Society of America. Clin Infect Dis 2009, 48:1003-1032. Evidence-based guidelines for the diagnosis and treatment of patients with influenza, published by the IDSA.

2. •- Guidance for clinicians on the use of rapid influenza diagnostic tests for the 2010-2011 influenza season. Available at http://www.cdc.gov/ $\mathrm{flu} / \mathrm{pdf} /$ professionals/diagnosis/clinician guidance ridt.pdf. Accessed October 2011. Most recent CDC guidelines for the use of rapid influenza diagnostic tests.

3. Monto AS, Gravenstein S, Elliott M, et al. Clinical signs and symptoms predicting influenza infection. Arch Intern Med. 2000;160:3243-7.

4. Boivin G, Hardy I, Tellier G, et al. Predicting influenza infections during epidemics with use of a clinical case definition. Clin Infect Dis. 2000;31:1166-9.

5. Call SA, Vollenweider MA, Hornung CA, et al. Does this patient have influenza? JAMA. 2005;293:987-97.

6. Dawood FS, Jain S, Finelli L, et al. Emergence of a novel swineorigin influenza A (H1N1) virus in humans. N Engl J Med. 2009;360:2605-15.

7. Bautista E, Chotpitayasunondh T, Gao Z, et al. Clinical aspects of pandemic 2009 influenza A (H1N1) virus infection. N Engl J Med. 2010;362:1708-19.

8. Belongia EA, Irving SA, Waring SC, et al. Clinical characteristics and 30-day outcomes for influenza A 2009 (H1N1), 2008-2009 (H1N1), and 2007-2008 (H3N2) infections. JAMA. 2010;304:1091-8.

9. Lee N, Chan PK, Lui GC, et al. Complications and outcomes of pandemic 2009 Influenza A (H1N1) virus infection in hospitalized adults: how do they differ from those in seasonal influenza? J Infect Dis. 2011;203:1739-47.
10. Kim EA, Lee KS, Primack SL, et al. Viral pneumonias in adults: radiologic and pathologic findings. Radiographics. 2002;22:S13749.

11. Peltola VT, Murti KG, McCullers JA. Influenza virus neuraminidase contributes to secondary bacterial pneumonia. J Infect Dis. 2005;192:249-57.

12. King QO, Lei B, Harmsen AG. Pneumococcal surface protein A contributes to secondary Streptococcus pneumoniae infection after influenza virus infection. J Infect Dis. 2009;200:537-45.

13. Wright PF, Kirkland KB, Modlin JF. When to consider the use of antibiotics in the treatment of $2009 \mathrm{H} 1 \mathrm{~N} 1$ influenza-associated pneumonia. N Engl J Med. 2009;361:e112.

14. Viasus D, Paño-Pardo JR, Pachón J, et al. Pneumonia complicating pandemic (H1N1) 2009: risk factors, clinical features, and outcomes. Medicine (Baltimore). 2011;90:328-36.

15. Kallen AJ, Brunkard J, Moore Z, et al. Staphylococcus aureus community-acquired pneumonia during the 2006 to 2007 influenza season. Ann Emerg Med. 2009;53:358-65.

16. Hageman JC, Uyeki TM, Francis JS, et al. Severe communityacquired pneumonia due to Staphylococcus aureus, 2003-04 influenza season. Emerg Infect Dis. 2006;12:894-9.

17. Centers for Disease Control and Prevention (CDC): Severe methicillin-resistant Staphylococcus aureus community-acquired pneumonia associated with influenza-Louisiana and Georgia, December 2006-January 2007. MMWR Morb Mortal Wkly Rep 2007, 56:325-329.

18. Hayashi Y, Vaska VL, Baba H, et al.: Influenza-associated Bacterial Pathogens in Patients with 2009 Influenza A (H1N1) Infection: Impact of Community-Associated Methicillin Resistant Staphylococcus aureus (MRSA) in Queensland, Australia. Intern Med J 2011, In press.

19. Randolph AG, Vaughn F, Sullivan R, et al. Critically Ill Children During the 2009-2010 Influenza Pandemic in the United States. Pediatrics. 2011; e1450-e1458

20. • Chowell G, Bertozzi SM, Colchero MA, et al.: Severe respiratory disease concurrent with the circulation of H1N1 influenza. N Engl J Med 2009, 361:674-679. An observational study in which the authors assessed the features on the 2009 swine influenza pandemic and found an increase in the rate of severe pneumonia and a shift in the age distribution of patients, compared to seasonal influenza cases.

21. • Perez-Padilla R, de la Rosa-Zamboni D, Ponce de Leon S, et al.: Pneumonia and respiratory failure from swine-origin influenza A (H1N1) in Mexico. N Engl J Med 2009, 361:680-689. Another observational study during the 2009 H1N1 influenza outbreak that supported the fact that this strain causes severe illness, ARDS and death in previously healthy persons who are young to middleaged.

22. • Brandão-Neto RA, Goulart AC, Santana AN, et al.: The role of Pneumonia scores in the emergency room in patients infected by 2009 H1N1 infection. Eur J Emerg Med 2011, In press. In this study, the authors prospectively assessed the utility of commonly used pneumonia severity scores in patients hospitalized for pandemic influenza A infection (H1N1). They found that both CURB65 and PSI were not accurate in predicting ICU admission.

23. Miller AC, Subramanian RA, Safi F, et al.: Influenza A 2009 (H1N1) Virus in Admitted and Critically Ill Patients. J Intensive Care Med 2011, In press.

24. Writing Committee of the Second World Health Organization Consultation on Clinical Aspects of Human Infection with Avian Influenza A (H5N1) Virus, Abdel-Ghafar AN, Chotpitayasunondh T, et al.: Update on avian influenza A (H5N1) virus infection in humans. N Engl J Med 2008, 358:261-273.

25. Booth S, Baleriola $\mathrm{C}$, Rawlinson WD. Comparison of two rapid influenza $\mathrm{A} / \mathrm{B}$ test kits with reference methods showing high specificity and sensitivity for influenza A infection. J Med Virol. 2006;78:619-22. 
26. Hurt AC, Alexander R, Hibbert J, et al. Performance of six influenza rapid tests in detecting human influenza in clinical specimens. J Clin Virol. 2007;39:132-5.

27. Smit M, Beynon KA, Murdoch DR, et al. Comparison of the NOW Influenza A \& B, NOW Flu A, NOW Flu B, and Directigen Flu A+B assays, and immunofluorescence with viral culture for the detection of influenza A and B viruses. Diagn Microbiol Infect Dis. 2007;57:67-70.

28. Rahman M, Vandermause MF, Kieke BA, et al. Performance of Binax NOW Flu A and B and direct fluorescent assay in comparison with a composite of viral culture or reverse transcription polymerase chain reaction for detection of influenza infection during the 2006 to 2007 season. Diagn Microbiol Infect Dis. 2008;62:162-6.

29. Centers for Disease Control and Prevention (CDC). Evaluation of rapid influenza diagnostic tests for detection of novel influenza $\mathrm{A}$ (H1N1) Virus - United States, 2009. MMWR Morb Mortal Wkly Rep 2009, 58:826-829.

30. Vasoo S, Stevens J, Singh K. Rapid antigen tests for diagnosis of pandemic (Swine) influenza A/H1N1. Clin Infect Dis. 2009;49:10903.

31. Drexler JF, Helmer A, Kirberg H, et al. Poor clinical sensitivity of rapid antigen test for influenza A pandemic (H1N1) 2009 virus. Emerg Infect Dis. 2009;15:1662-4.

32. Pongthanapisith V, Sukasem C, Premchaiporn K, et al. Clinical performance of three rapid diagnostic tests for influenza virus in nasopharyngeal specimens to detect novel swine-origin influenza viruses. Infection. 2011;39:105-11.

33. Landry ML, Cohen S, Ferguson D. Real-time PCR compared to Binax NOW and cytospin-immunofluorescence for detection of influenza in hospitalized patients. J Clin Virol. 2008;43:148-51.

34. Taubenberger JK, Layne SP. Diagnosis of influenza virus: coming to grips with the molecular era. Mol Diagn. 2001;6:291-305.

35. Ellis JS, Zambon MC. Molecular diagnosis of influenza. Rev Med Virol. 2002;12:375-89.

36. - Shu B, Wu KH, Emery S, et al.: Design and performance of the CDC real-time reverse transcriptase PCR swine flu panel for detection of 2009 A (H1N1) pandemic influenza virus. J Clin Microbiol 2011, 49:2614-2619. Description of the rRT-PCR assay designed at the CDC during the $2009 \mathrm{H} 1 \mathrm{~N} 1$ influenza A pandemic.

37. Yang Y, Huang F, Gonzalez R, et al. Evaluation of twelve real-time reverse transcriptase $\mathrm{PCR}$ primer-probe sets for detection of pandemic influenza A/H1N1 2009 virus. J Clin Microbiol. 2011;49:1434-40.

38. Lam WY, Leung TF, Lee N, et al. Development and comparison of molecular assays for the rapid detection of the pandemic influenza A (H1N1) 2009 virus. J Med Virol. 2010;82:675-83.

39. de la Tabla VO, Masiá M, Antequera P, et al. Comparison of combined nose-throat swabs with nasopharyngeal aspirates for detection of pandemic influenza A/H1N1 2009 virus by real-time reverse transcriptase PCR. J Clin Microbiol. 2010;48:3492-5.

40. •- Centers for Disease Control and Prevention (CDC): Prevention and control of influenza with vaccines: recommendations of the Advisory Committee on Immunization Practices (ACIP), 2011. MMWR Morb Mortal Wkly Rep 2011, 60:1128-1132. Latest recommendations for influenza vaccination from the CDC.

41. •• Fiore AE, Fry A, Shay D, et al.: Antiviral agents for the treatment and chemoprophylaxis of influenza - recommendations of the Advisory Committee on Immunization Practices (ACIP). MMWR Recomm Rep 2011,60:1-24. ACIP recommendations on the use of antiviral medications for the treatment and prevention of influenza.

42. Toovey S, Rayner C, Prinssen E, et al. Assessment of neuropsychiatric adverse events in influenza patients treated with oseltamivir: a comprehensive review. Drug Saf. 2008;31:1097-114.

43. Tanabe T, Hara K, Nakajima M, et al. Oseltamivir treatment for children showing abnormal behavior during influenza virus infection. Brain Dev. 2010;32:440-4.
44. Williamson JC, Pegram PS. Neuraminidase inhibitors in patients with underlying airways disease. Am J Respir Med. 2002;1:8590.

45. Keyser LA, Karl M, Nafziger AN, et al. Comparison of central nervous system adverse effects of amantadine and rimantadine used as sequential prophylaxis of influenza A in elderly nursing home patients. Arch Intern Med. 2000;160:1485-8.

46. Cooper NJ, Sutton AJ, Abrams KR, et al. Effectiveness of neuraminidase inhibitors in treatment and prevention of influenza $A$ and B: systematic review and meta-analyses of randomised controlled trials. BMJ. 2003;326:1235.

47. Jefferson T, Demicheli V, Rivetti D, et al. Antivirals for influenza in healthy adults: systematic review. Lancet. 2006;367:303-13.

48. Jefferson T, Jones M, Doshi P, et al. Neuraminidase inhibitors for preventing and treating influenza in healthy adults: systematic review and meta-analysis. BMJ. 2009;339:b5106.

49. Burch J, Corbett M, Stock C, et al. Prescription of anti-influenza drugs for healthy adults: a systematic review and meta-analysis. Lancet Infect Dis. 2009;9:537-45.

50. Randomised trial of efficacy and safety of inhaled zanamivir in treatment of influenza A and B virus infections. The MIST (Management of Influenza in the Southern Hemisphere Trialists) Study Group. Lancet 1998, 352:1877-1881.

51. Kaiser L, Wat C, Mills T, et al. Impact of oseltamivir treatment on influenza-related lower respiratory tract complications and hospitalizations. Arch Intern Med. 2003;163:1667-72.

52. Hernán MA, Lipsitch M. Oseltamivir and risk of lower respiratory tract complications in patients with flu symptoms: a meta-analysis of eleven randomized clinical trials. Clin Infect Dis. 2011;53:277-9.

53. Lee N, Chan PK, Choi KW, et al. Factors associated with early hospital discharge of adult influenza patients. Antivir Ther. 2007;12:501-8.

54. McGeer A, Green KA, Plevneshi A, et al. Antiviral therapy and outcomes of influenza requiring hospitalization in Ontario, Canada. Clin Infect Dis. 2007;45:1568-75.

55. Uyeki T. Antiviral treatment for patients hospitalized with 2009 pandemic influenza A (H1N1). N Engl J Med. 2009;361:e110.

56. Jain S, Kamimoto L, Bramley AM, et al. Hospitalized patients with 2009 H1N1 influenza in the United States, April-June 2009. N Engl J Med. 2009;361:1935-44.

57. Rodríguez A, Díaz E, Martín-Loeches I, et al. Impact of early oseltamivir treatment on outcome in critically ill patients with 2009 pandemic influenza A. J Antimicrob Chemother. 2011;66:1140-9.

58. Yu H, Liao Q, Yuan Y, et al. Effectiveness of oseltamivir on disease progression and viral RNA shedding in patients with mild pandemic 2009 influenza A H1N1: opportunistic retrospective study of medical charts in China. BMJ. 2010;341:c4779.

59. Choi SM, Boudreault AA, Xie H, et al. Differences in clinical outcomes after 2009 influenza A/H1N1 and seasonal influenza among hematopoietic cell transplant recipients. Blood. 2011;117:5050-6.

60. Gaur $\mathrm{AH}$, Bagga $\mathrm{B}$, Barman $\mathrm{S}$, et al. Intravenous zanamivir for oseltamivir-resistant 2009 H1N1 influenza. N Engl J Med. 2010;362:88-9.

61. Kidd IM, Down J, Nastouli E, et al. H1N1 pneumonitis treated with intravenous zanamivir. Lancet. 2009;374:1036.

62. Härter G, Zimmermann O, Maier L, et al. Intravenous zanamivir for patients with pneumonitis due to pandemic (H1N1) 2009 influenza virus. Clin Infect Dis. 2010;50:1249-51.

63. Dulek DE, Williams JV, Creech CB, et al. Use of intravenous zanamivir after development of oseltamivir resistance in a critically Ill immunosuppressed child infected with 2009 pandemic influenza A (H1N1) virus. Clin Infect Dis. 2010;50:14936.

64. Kohno S, Kida H, Mizuguchi M, et al. Intravenous peramivir for treatment of influenza A and B virus infection in high-risk patients. Antimicrob Agents Chemother. 2011;55:2803-12. 
65. Kohno S, Kida H, Mizuguchi M, et al. Efficacy and safety of intravenous peramivir for treatment of seasonal influenza virus infection. Antimicrob Agents Chemother. 2010;54:456874.

66. Watanabe A, Chang SC, Kim MJ, et al. Long-acting neuraminidase inhibitor laninamivir octanoate versus oseltamivir for treatment of influenza: A double-blind, randomized, noninferiority clinical trial. Clin Infect Dis. 2010;51:1167-75.

67. Kandun IN, Tresnaningsih E, Purba WH, et al. Factors associated with case fatality of human $\mathrm{H} 5 \mathrm{~N} 1$ virus infections in Indonesia: a case series. Lancet. 2008;372:744-9.

68. Liem NT, Tung CV, Hien ND, et al. Clinical features of human influenza A (H5N1) infection in Vietnam: 2004-2006. Clin Infect Dis. 2009;48:1639-46.

69. WHO. Clinical management of human infection with avian influenza A (H5N1) virus (15 August 2007). Available at http://www.who.int/influ enza/resources/documents/clinical_management_h5n1_15_08_2007/en/ index.html. Accessed: November 2011.

70. • Centers for Disease Control and Prevention. 2011-2012 influenza antiviral medications: summary for clinicians. Available at http:// www.cdc.gov/flu/professionals/antivirals/summary-clinicians.htm. Accessed November 2011. CDC guidelines for the use of antiviral medications for the treatment and prevention of influenza.
71. Hurt AC, Lee RT, Leang SK, et al.: Increased detection in Australia and Singapore of a novel influenza A(H1N1)2009 variant with reduced oseltamivir and zanamivir sensitivity due to a S247N neuraminidase mutation. Euro Surveill 2011, 16.

72. Hayden FG, de Jong MD. Emerging influenza antiviral resistance threats. J Infect Dis. 2011;203:6-10.

73. Mandell LA, Wunderink RG, Anzueto A, et al. Infectious Diseases Society of America/American Thoracic Society consensus guidelines on the management of community-acquired pneumonia in adults. Clin Infect Dis. 2007;44:S27-72.

74. - Kumar A, Zarychanski R, Pinto R, et al.: Critically ill patients with 2009 influenza A(H1N1) infection in Canada. JAMA 2009, 302:1872-1879. Canadian prospective study which showed that, during the H1N1 influenza A pandemic, lower respiratory tract compromise was rapidly progressive, oftenly leading to respiratory failure and requiring salvage therapies.

75. Noah MA, Peek GJ, Finney SJ, et al. Referral to an extracorporeal membrane oxygenation center and mortality among patients with severe 2009 influenza A(H1N1). JAMA. 2011;306:1659-68.

76. Dellinger RP, Levy MM, Carlet JM, et al. Surviving Sepsis Campaign: international guidelines for management of severe sepsis and septic shock: 2008. Crit Care Med. 2008;36:296-327. 